\title{
Association of cigarette smoking with irritable bowel syndrome: A cross-sectional study
}

\author{
Khalid Mahmood ${ }^{1}$, Ramsha Riaz ${ }^{2}$, Muhammad Salman UI Haq ${ }^{* 2}$ (D) Khizar Hamid ${ }^{2}$, Hassaan Jawed ${ }^{2}$ \\ Received: 18 Mar 2019 \\ Published: 1 Jul 2020
}

\section{Abstract}

Background: Irritable Bowel Syndrome (IBS) is one of the most commonly diagnosed gastrointestinal disorders, and its etiology is believed to be multifactorial. The role of smoking in the pathophysiology of IBS still remains inconclusive. Hence, we aim to investigate whether or not an association exists between smoking and IBS.

Methods: A cross-sectional study was conducted in a tertiary care hospital of Karachi. A sample size of 200 smokers and 200 nonsmokers was selected. A two-part self-reported questionnaire was administered to the participants and the diagnosis of IBS was based on the Rome III diagnostic criteria. All data were analyzed using the Statistical Package for the Social Sciences version 17. Statistical tests employed were Independent samples t-test, chi-square test, and binary logistic regression analysis to obtain Odds Ratio (OR) for various variables. A P-value $<0.05$ was considered statistically significant.

Results: Overall prevalence of IBS based on Rome III criteria was 9.5\% (95\% CI 9.45-9.60). Smoking was significantly found to be associated with IBS $(\mathrm{P}=0.002)$. Binary logistic regression analysis indicated that non-smokers were more likely to have IBS (Crude OR: $3.00,95 \%$ CI 1.23-7.30) than smokers, and females were more likely to have IBS (Crude OR: $3.73,95 \%$ CI 1.56-7.69) than males. Age, BMI, and food groups were not found to be significantly associated with IBS.

Conclusion: Our study concludes that IBS was more prevalent among non-smokers and amongst females. Further studies are required to firmly establish the role of smoking in IBS.

Keywords: Irritable bowel syndrome, Smoking, Risk factors, Gastrointestinal diseases, Pakistan

Conflicts of Interest: None declared

Funding: None

\section{*This work has been published under CC BY-NC-SA 1.0 license. \\ Copyright $\subseteq$ Iran University of Medical Sciences}

Cite this article as: Mahmood K, Riaz R, Ul Haq MS, Hamid K, Jawed H. Association of cigarette smoking with irritable bowel syndrome: A crosssectional study. Med J Islam Repub Iran. 2020 (1 Jul);34:72. https://doi.org/10.47176/mjiri.34.72

\section{Introduction}

Irritable bowel syndrome (IBS) is a functional gastrointestinal disorder characterized by abdominal pain/discomfort and disturbed bowel action (1). The term 'functional' signifies the absence of demonstrable histological or anatomical pathology and explains IBS in terms of a symptom

Corresponding author: Dr Muhammad Salman UI Haq, m.salman.haq@gmail.com

1. Department of Internal Medicine, Dr. Ruth Pfau Civil Hospital Karachi, Baba-e-Urdu Road, Karachi, Pakistan

2. Dow Medical College, Dow University of Health Sciences, Baba-e-Urdu Road, Karachi, Pakistan complex (2). It is one of the most common gastrointestinal conditions diagnosed by general practitioners and affects approximately $11 \%$ of the population around the globe (3).

The diagnosis of IBS is base $\mathrm{d}$ on clinical criteria that

$\uparrow$ What is "already known" in this topic:

It has been well documented that IBS is associated with multiple factors such as anxiety, depression, mental and psychological stress, analgesics, and food intake, but as of yet, no such association has been established with smoking. Furthermore, smoking has been implicated in other gastrointestinal disorders, such as ulcerative colitis and Crohn's disease.

\section{$\rightarrow$ What this article adds:}

In this research, we have concluded that IBS does have a statistically significant association with smoking. IBS is more prevalent in non-smokers as compared to smokers and it is also more prevalent in females as compared to males. 
have changed over time (4). Three main sets of diagnostic criteria have been employed over the years; Manning criteria, Rome I criteria, and the later Rome II criteria (1). Manning criteria seems to yield higher values when compared to either the Rome I or Rome II criteria; however, in current practice, the Rome III criteria is being used more extensively (5).

As a result of the different diagnostic criteria employed in different studies, significant heterogeneity exists amongst available literature, and the percentage prevalence of IBS varies immensely internationally (3). In the Western populations, based on the Rome III diagnostic criteria, the prevalence of IBS can range from $16 \%(6,7)$ to $29.16 \%$ (8). Though IBS is a relatively common functional disorder in the West, data on its prevalence amongst the Asian population is comparatively scanty, with an exceedingly variable range of prevalence i.e. $2.3 \%$ to $34 \%$ (5). Similarly, limited data is available on the Pakistani population. Several studies aiming to investigate the frequency of IBS amongst our population have reported a prevalence of $28.3 \%$ (5) based on the Rome III criteria, and $13.3 \%$ to as high as $45 \%(9,10)$ based on older criterion.

Regardless of IBS being significantly prevalent in the general population, there is a restricted understanding of its causes, pathogenesis, and treatment. The etiology of IBS is not properly understood, but is attributed to be multifactorial in nature (11). Several studies have delineated the significant role of anxiety, depression, mental and psychological stress, and physical and sexual abuse as contributing factors in the irritable bowel syndrome $(2,5$, $11)$. IBS has also been found to be significantly associated with the use of certain analgesics (12), with food intake $(2,12)$, and with acute infectious gastroenteritis leading to post-infectious IBS (11). Furthermore, a review article suggests that genetic factors may also play a role in IBS; however, data regarding this still remains ambiguous and unsatisfactory (3).

The role of tobacco smoking has been strongly implicated in a number of pathologies relating to the gastrointestinal tract. Smoking has been well known to provide a protective and beneficial effect on ulcerative colitis (13, 14), but acts as a major risk factor for various other disorders including Crohn's disease, peptic ulcer disease, Gastroesophageal reflux disease (GERD), and gastric carcinoma (14). In contrast to this, the effect of smoking in regard to irritable bowel syndrome has not been clearly established so far. While it has been suggested that smoking may have a protective role in irritable bowel syndrome as well (13), data defining such a relationship remains scarce and largely inconclusive.

In a country like Pakistan where smoking is widespread, with an average national prevalence of $21.6 \%$ (36\% males and $9 \%$ females) (15), it is imperative to explore any role that it may have in the course of a disease. Moreover, with IBS being so prevalent, it not only exerts a detrimental impact on the quality of life of an individual but also places considerable financial constraints on the patient and an economic burden on the health care sector of a country (16). This further emphasizes the need to investigate un- known factors that may be related to this disorder. Given the paucity of information on this subject, the primary objective of our research is to investigate whether or not an association occurs between smoking and IBS. Furthermore, our secondary objective is to estimate the frequency of IBS in our population and to find out if it is related to a number of other factors such as gender, age, body mass index (BMI), and dietary pattern. Hence, the null hypothesis for our research states that there exists no association of smoking with IBS, while our alternate hypothesis states a possible association of smoking and IBS. With a better understanding of the epidemiology and risk factors of the disease, the physician will be able to provide effective counselling and exercise a more accurate management plan for affected individuals.

\section{Methods}

This cross-sectional study was conducted in a tertiary care hospital of Pakistan and its affiliated medical college, over a period of five months from March 2018 to July 2018. The sample size was calculated using Open Epi, taking $\mathrm{p}$ as $28.3 \%$ (5) at $95 \%$ Confidence Interval (CI). A sample size of 312 was obtained; however, to increase the accuracy of our results, a sample of 400 was selected. To assess the relationship between smoking and IBS, the sample was equally divided into 200 non-smokers and 200 smokers. The convenience sampling method was employed to recruit students and faculty members from the medical college, and medical staff and patient's attendants from the hospital. To exclude possible organic disease, a list of exclusion criteria based on red flag symptoms was drawn out, and individuals having any of the symptoms (based on history) from the following list were not included in our study: fever; significant unintentional weight loss; episodes of per rectal bleeding; any swelling or a lump in the abdomen or rectal/anal area; known iron deficiency anemia; family history of colon cancer or celiac disease; nausea, recurrent vomiting, or diarrhea that is persistent or awakens them from sleep; abdominal pain not completely relieved by a bowel movement, or occurring at night.

Upon being approached, individuals were first inquired about each of these symptoms by the interviewers. After having ensured the absence of these symptoms, written informed consent was obtained from each participant, and they were explained the purpose of our research and assured regarding the confidentiality of their data. Subjects were grouped according to their smoking status. An individual was considered to be a smoker if he/she consumed a minimum of one cigarette per day for a period of at least six months. The research abided by the ethical guidelines of the declaration of Helsinki.

A structured, self-reported questionnaire was administered to the participants, which comprised two parts. The first part covered general demographic variables concerning age, gender, height, weight, smoking status, duration of smoking, and the number of cigarettes consumed per day. Height and weight were used to calculate the BMI of the participants by dividing the weight (in kilograms) by the square of height (in centimeters). For quantification of 
smoking, the product of duration of smoking and number of cigarettes consumed each day was divided by 20 , in order to calculate the pack years. Furthermore, this section also assessed the participants for any food groups that trigger abdominal discomfort, the use of medications for GI complaints, as well as their health-seeking behavior such as visits to the physician for similar complaints. The second portion of the questionnaire was based on the Rome III criteria for the diagnosis of IBS. A subject was considered to be IBS positive if he/she was suffering from recurrent abdominal pain/discomfort at least three days per month in the last three months, with symptom onset at least six months prior to diagnosis, along with two or more of the following: (i) Improvement/relief of pain or discomfort with defecation; (ii) Onset of pain or discomfort associated with a change in frequency of stool (either more stools or fewer stools); (iii) Onset of pain or discomfort associated with a change in consistency/appearance of stool (either looser stools or harder stools).

IBS positive individuals were further sub-classified according to the types of IBS as having constipation predominant, diarrhea predominant, mixed, or unsub-typed IBS.

In cases where the participant could not read, write, or understand English, the questionnaire was translated to the subjects in the language they understood, and their responses were recorded by the interviewer. In order to minimize interviewer bias in these circumstances, the interviewers followed the same code of conduct in terms of body language, mannerism and speech.

All data were analyzed using the Statistical Package for the Social Sciences (SPSS) v17. An independent sample ttest was performed for continuous variables, while chisquare test was used to assess whether or not an association occurs between smoking and Irritable Bowel Syndrome. A P-value $<0.05$ was considered statistically significant. Characteristics and subtypes of IBS, along with food groups triggering abdominal discomfort and healthseeking behavior of participants, were presented in the form of frequencies and percentages. Furthermore, binary logistic regression analysis was performed to calculate the P-values, Odds Ratio (OR) and 95\% confidence intervals (CI) for various independent variables such as smoking status, pack-years, gender, age, and food groups. Adjusted Odds Ratio (Adj OR) for smoking was also calculated using the main food groups triggering abdominal discomfort (meat, dairy, fried, and spicy food categories), age, and BMI as covariates, to eliminate their possible influence on the association between smoking and IBS. Similarly, adj OR for all other variables was also obtained us- ing the rest of the variables as covariates.

\section{Results}

A total of 200 smokers and an equal number of nonsmokers were recruited in our study. The majority of the participants from both the groups were males $(n=289$, $\sim 72 \%$ ), while females comprised approximately $28 \%$ $(n=111)$ of the study population. Smokers had a higher mean age (37.4 \pm 13.0 years) and a higher mean BMI $(23.2 \pm 4.1 \mathrm{~kg} / \mathrm{m} 2)$ as compared to non-smokers, however as opposed to the difference in age $(\mathrm{P}<0.001)$, the difference in BMI was not statistically significant (Table 1).

Out of the total sample, $9.5 \%$ (95\% CI 9.45-9.60) of the participants were diagnosed with IBS based on the Rome III criteria, indicating that approximately 1 in 10 people suffer from this disease. More than two-third of the people diagnosed with IBS were non-smokers $(28 / 38,73.7 \%)$, as compared to less than half this number of smokers $(10 / 38$, $26.3 \%$ ). Chi-square test revealed a statistically significant association between smoking and the incidence of IBS $(\mathrm{P}=0.002)$. Furthermore, the overall percentage of females being affected by IBS was slightly greater than the percentage of affected men (21/38, 55.3\% females; $17 / 38$, $44.7 \%$ males). Smokers with IBS were reported to have a higher mean age (39.9 years) as compared to their nonsmoker counterparts (31.8 years). The most common subtype of IBS seen in non-smokers was IBS-M $(12 / 28$, $42.9 \%)$, followed by IBS-D $(7 / 28,25 \%)$, IBS-C $(5 / 28$, $17.9 \%$ ), and lastly, IBS-U being the least common variety $(4 / 28,14.3 \%)$. In smokers however, both IBS-M and IBS$\mathrm{D}$ were the predominant sub-types, occurring in equal incidence (4/10, 40\% each). Abdominal pain/discomfort was the most frequent complaint experienced by all of the participants $(38 / 38,100 \%)$ diagnosed with IBS in both groups. Improvement with defecation also occurred in all of the smokers with IBS $(10 / 10,100 \%)$, but was reported by only $3 / 4$ th of the non-smokers with IBS $(21 / 28,75 \%)$. (Table 2).

Binary logistic regression depicts the association of a number of independent variables to IBS. The crude odds ratio for individuals with a positive smoking status indicates a reduced incidence of IBS (Crude OR: 0.33, 95\% CI 0.14-0.81), while non-smokers are more likely to have IBS than smokers (Crude OR: 3.00, 95\% CI 1.23-7.30). After adjusting for some of the major food groups precipitating abdominal discomfort in individuals, the adjusted odds ratio remained largely unchanged (Adj OR: 0.33, 95\% CI 0.13-0.80; Adj OR: 3.03, 95\% CI 1.24-7.44 respectively). Another important association observed is between gender and IBS. Males are less likely to have IBS

\begin{tabular}{|c|c|c|c|}
\hline Variable & Non-smokers $(n=200)$ & Smokers $(n=200)$ & $\mathrm{P}_{\text {-value }}{ }^{\mathrm{a}}$ \\
\hline \multicolumn{4}{|l|}{ Gender } \\
\hline Male & 116 & 173 & $<0.001$ \\
\hline Female & 84 & 27 & \\
\hline Mean BMI $\left(\mathrm{kg} / \mathrm{m}^{2}\right) \pm \mathrm{SD}$ & $22.7 \pm 5.8$ & $23.2 \pm 4.1$ & 0.368 \\
\hline Mean Age (years) \pm SD & $28.5 \pm 11.1$ & $37.4 \pm 13.0$ & $<0.000$ \\
\hline
\end{tabular}




\begin{tabular}{|c|c|c|c|}
\hline Variable & Non-smokers $(n=200)$ & Smokers $(n=200)$ & P-value ${ }^{b}$ \\
\hline Diagnosed with IBS (\%) & $28(14 \%)$ & $10(5 \%)$ & 0.002 \\
\hline Males & $12(6 \%)$ & $5(2.5 \%)$ & \\
\hline Females & $16(8 \%)$ & $5(2.5 \%)$ & \\
\hline Mean age in years (range) & $31.8(18-60)$ & $39.9(23-50)$ & \\
\hline Sub-types of IBS & & & 0.806 \\
\hline IBS-C & 5 & 1 & \\
\hline IBS-D & 7 & 4 & \\
\hline IBS-M & 12 & 4 & \\
\hline IBS-U & 4 & 1 & \\
\hline Symptoms (\%) & & & 0.872 \\
\hline Abdominal pain/discomfort & $28(100 \%)$ & $10(100 \%)$ & \\
\hline Improvement with defecation & $21(75 \%)$ & $10(100 \%)$ & \\
\hline Change in frequency of stool & $21(75 \%)$ & $7(70 \%)$ & \\
\hline Change in consistency of stool & $23(82 \%)$ & $7(70 \%)$ & \\
\hline \multicolumn{4}{|c|}{$\begin{array}{l}\text { IBS: Irritable Bowel Syndrome; IBS-C: constipation-predominant; IBS-D: diarrhea-predominant; IBS-M: mixed; IBS-U: unsubtyped. } \\
\text { b Calculated using chi-square; p-value }<0.05 \text { considered statistically significant }\end{array}$} \\
\hline \multicolumn{4}{|c|}{ Table 3. Odds Ratio for Irritable Bowel Syndrome for various independent variables } \\
\hline Variable & P-value ${ }^{c}$ & Crude OR $(95 \% \mathrm{CI})$ & Adjusted OR $(95 \% \mathrm{CI})$ \\
\hline \multicolumn{4}{|l|}{ Smoking Status } \\
\hline Yes & 0.015 & $0.33(0.14-0.81)$ & $0.33(0.13-0.80)$ \\
\hline No & & $3.00(1.23-7.30)$ & $3.03(1.24-7.44)$ \\
\hline \multicolumn{4}{|l|}{ Duration of Smoking* } \\
\hline Less than 10 pack-years & 0.450 & $1.91(0.36-10.22)$ & $1.94(0.39-10.25)$ \\
\hline Greater than 10 pack-years & 0.450 & $0.52(0.10-2.80)$ & $0.55(0.13-2.83)$ \\
\hline \multicolumn{4}{|l|}{ Food Groups } \\
\hline Meat products & 0.344 & $0.61(0.21-1.71)$ & $0.57(0.17-1.80)$ \\
\hline Dairy products & 0.974 & $0.98(0.21-4.44)$ & $1.13(0.23-5.64)$ \\
\hline Fried Food & 0.103 & $0.51(0.23-1.15)$ & $0.96(0.31-2.93)$ \\
\hline Spicy Food & 0.162 & $0.57(0.26-1.25)$ & $0.72(0.25-2.07)$ \\
\hline \multicolumn{4}{|l|}{ Gender } \\
\hline Male & 0.000 & $0.28(0.13-0.64)$ & $0.26(0.10-0.69)$ \\
\hline Female & & $3.57(1.56-7.69)$ & $3.55(1.54-7.71)$ \\
\hline Age (years) & 0.982 & $1.00(0.97-1.03)$ & $1.03(0.99-1.07)$ \\
\hline BMI $\left(\mathrm{kg} / \mathrm{m}^{2}\right)$ & 0.163 & $0.94(0.85-1.03)$ & $0.95(0.87-1.04)$ \\
\hline
\end{tabular}

(OR: 0.27 , 95\% CI 0.12-0.62), while females are more likely to be diagnosed with the disease (OR: $3.73,95 \%$ CI 1.63-8.03). The odds ratios calculated for other variables are not statistically significant (Table 3 ).

Amongst the various medications/remedies used by participants for gastrointestinal complaints, the use of Ispaghol/Ispaghula (psyllium seed husk) was the most popular choice $(106 / 400,26.5 \%)$. Only a small percentage of individuals with IBS $(5 / 38,13.2 \%)$ had paid a visit to a physician regarding their GI complaints. None of the individuals diagnosed with IBS in our study were previously diagnosed with this disease by a physician (Table 4).

The most common foods triggering abdominal discomfort/pain were spicy foods and fried foods $(31.75 \%$ and $26 \%$ respectively), followed by meat products $(12.25 \%)$, with all three of them together accounting for $70 \%$ of the food related GI complaints (Fig. 1).

\section{Discussion}

Our study was primarily conducted with the aim of delineating a possible relationship between smoking and IBS. The results of our study support a relationship between smoking and Irritable Bowel Syndrome. Our findings indicate that the prevalence of IBS is significantly less in the smoker population, whereas the non-smoker population has an increased incidence of IBS. There is a considerable lack of data that investigates and supports such a relationship, however, one of the oldest researches to find a possible association between smoking and IBS was carried out by Burns (1986), where he postulated that the protective role of smoking in ulcerative colitis might also be applicable to IBS, since there were a higher num-

Table 4. Use of medications and health-seeking behavior amongst participants

\begin{tabular}{|c|c|c|c|}
\hline Use of medications for GI complains (\%) & $\begin{array}{c}\text { IBS Positive } \\
(n=38)\end{array}$ & $\begin{array}{c}\text { IBS Negative } \\
(\mathrm{n}=362)\end{array}$ & $\begin{array}{c}\text { Total } \\
(n=400)\end{array}$ \\
\hline Ispaghol/ispaghula (psyllium seed husk) & 11 & 95 & $106(26.5 \%)$ \\
\hline Anti-spasmodic & 1 & 0 & $1(0.25 \%)$ \\
\hline Anti-diarrheal & 3 & 2 & $5(1.25 \%)$ \\
\hline Metronidazole & 3 & 11 & $14(3.5 \%)$ \\
\hline Antacid & 5 & 15 & $20(5 \%)$ \\
\hline Consulted physician for GI complains (\%) & 5 & 15 & $20(5 \%)$ \\
\hline Previously diagnosed with IBS by physician (\%) & 0 & 0 & $0(0 \%)$ \\
\hline
\end{tabular}

IBS: I 


\section{IBS positive}

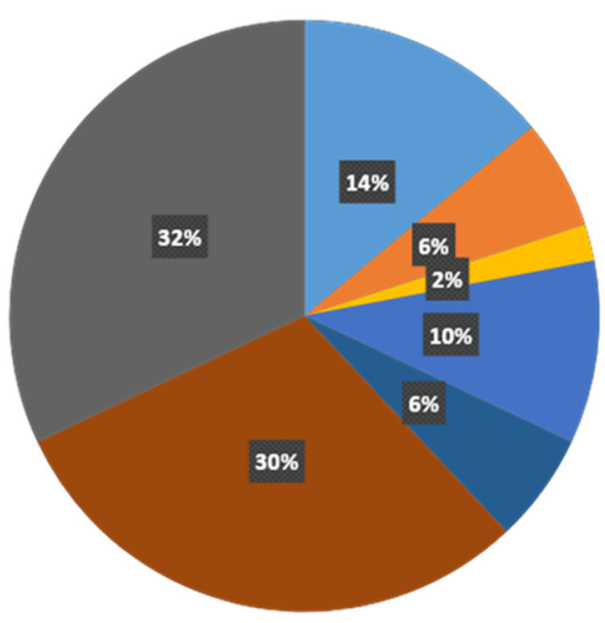

Fig. 1. Foods triggering abdominal discomfort

ber of non-smokers in the IBS group of his study (13). Very little research has been carried out since then to establish the role of smoking in the course of this disease. Several studies have reported no correlation of smoking status with IBS $(12,17)$. Similarly, another study conducted in Syria reported cigarette smoking to be significantly associated with dyspepsia and GERD, but not with IBS (18). Moreover, another study found only female nonsmokers to be at an increased risk of developing IBS (OR $6.19, \mathrm{P}=0.04$ ), but reported no overall correlation of smoking habit with IBS (19).

Given that the etiology of IBS is still not fully understood, it is difficult to assess why smoking led to a decreased incidence of IBS in our study population. Studies have been conducted to investigate the effects of smoking on gut mucosa (20), gut motility (21), and stool consistency (22), one of which may serve to explain the possible mechanism behind our findings. A study conducted on mice gut demonstrated that the components found in cigarette smoke led to an alteration in the composition of gut microflora, and reduced mucosal inflammation, associated with an increased expression of tight junction proteins (20). Furthermore, smoking was also found to decrease gut motility by prolonging the mouth-cecum transit time (21). In addition, a study investigating the role of various foods and beverages on stool consistency reported that cigarettes acted as a stool softener in half of the smokers who had constipation-predominant IBS (22). While these studies may not fully explain our findings, they suggest that certain components of cigarette smoke may be responsible for affecting the gut and /or gut mucosa and preventing the development of IBS. In order to fully elucidate this unusual effect, future research pertaining to this topic is warranted, which may pave the way for the devel-

\section{IBS Negative}

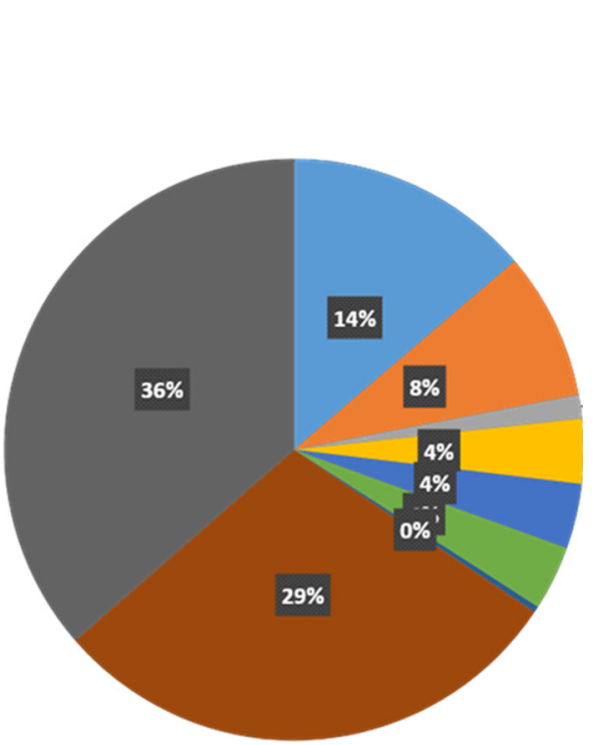

opment of appropriate therapeutic modalities for this disease and help physicians in understanding this pathology better.

Furthermore, this study also reports that the overall prevalence of IBS in our study population is $9.5 \%$, with females being affected slightly more than men. A number of studies conducted in Pakistan have reported a prevalence of IBS higher than that reported in our study. A study on medical students employing the Rome III criteria reported a prevalence of $28.3 \%$, with females being predominantly affected (5). Another study based on college students revealed a frequency of 34\% (23). A study set in two cities of Pakistan, Karachi, and Bahawalpur, revealed an overall prevalence rate of $45 \%$; however, in contrast to our study, the frequency of men suffering from IBS was greater than that of women (10). The wide disparity in the frequencies investigated by various researches may be due to different diagnostic criteria being employed, and the studies being based on different groups of people. In addition, gender was found to be the only variable besides smoking to be significantly associated with IBS in our study, with the female gender at an increased likelihood to have IBS $(\mathrm{OR}=3.73)$, as compared to males $(\mathrm{OR}=0.27)$. These results are concordant with a study that reported that the female gender is a significant risk factor for IBS (18). Internationally, IBS is predominantly found to affect women as well (16). Moreover, a meta-analysis also explains that irritable bowel syndrome is more common in women, possibly due to the interplay of female hormones or other psychological differences between men and women (24). On the other hand, another research found no association between gender and IBS (12). The impact of gender on IBS is clinically relevant, because the treatment response to certain medications differs in both the genders 
(24), and it has been reported that women tend to benefit more from the use of 5-HT3 receptor antagonists as compared to men (25). Hence, understanding and reporting gender differences would assist clinicians in prescribing appropriate therapies accordingly.

Apart from smoking and gender, our study reports no significant association between IBS, age, and BMI. A study set in Iran also found no relationship between BMI and IBS, but reported IBS to be associated with younger age (17). On the contrary, another study concluded that IBS was associated with a lower BMI in females exclusively, while no association was found with age (19). Moreover, based on our study, the most common food precipitating abdominal symptoms was found to be spicy and fried food. This vastly differs from the foods causing abdominal symptoms in a western country, where most people reported abdominal pain from eating foods containing carbohydrates and fats (26). Cultural differences in cuisine may account for such distinction, but this contrast is significant because different diet plans will need to be implemented in different countries according to the major type of food causing symptom exacerbations, as part of a precautionary management plan.

Another important finding revealed by this study was the low frequency of visits to the physician regarding GI complaints. Out of all the people diagnosed with IBS, only $13.2 \%$ had consulted a physician, while none of them had been diagnosed with IBS previously. This either reflects a lack of knowledge and awareness regarding IBS amongst the population, or demonstrates a general hesitancy amongst people in visiting a doctor for what they perceive to be 'minor complaints'. As opposed to these findings, another study based on the Pakistani population reported $60 \%$ of individuals with IBS seeking health care advice, with men constituting the majority (10). Differences in educational and socioeconomic backgrounds amongst participants of the two studies may have accounted for this disparity in health-seeking behavior. Nevertheless, this is a significant finding with valuable clinical implications. The health-related quality of life of people suffering from IBS is generally poor (27), and proper therapeutic intervention owing to timely consultations with a physician can alleviate this, allowing these patients to lead a better life. This emphasizes the importance of promoting awareness regarding relatively common disorders like IBS amongst the population and discouraging the concept of self-medication for seemingly trivial complaints.

While our study has presented valuable findings pertaining to IBS in our population, it has some limitations. Firstly, the study was conducted in just one hospital and medical college of the city, making it difficult to generalize the findings to a larger population. Secondly, the study sample was not randomized, and the two groups of subjects were not matched based on gender and age. Thirdly, we did not investigate the role of some other factors causing IBS such as stress, anxiety, and depression, which may have acted as confounders. Moreover, the quality of life of individuals with and without IBS was neither measured nor compared. Our study was based on a self-reporting tool, which is subjective in nature, and may have led to recall bias. Furthermore, participants were subjectively questioned regarding the list of exclusion criteria, and no lab investigations/clinical examinations were carried out. Also, individuals who may have been exposed to passive smoking of varying degrees were not accounted for.

\section{Conclusion}

The results of our study indicate that the prevalence of IBS based on the Rome III diagnostic criteria was $9.5 \%$. IBS was found to be associated with smoking and female gender. There was no association of IBS with pack-years of smoking, food groups, age, and BMI. We also highlighted the low frequency of health-seeking behavior amongst participants, exhibiting the need for better awareness regarding the pathology for an improved health-related quality of life. Future, larger-scale researches based on different communities are required to accurately delineate the role of smoking in IBS, which may pave the way for the development of newer treatment options and management plans for people suffering from this disorder.

\section{Acknowledgments}

The authors would like to thank all the anonymous reviewers for their comments and suggestions, which were extremely insightful. All authors were involved in the collection of data and the writing of the manuscript.

\section{Conflict of Interests}

The authors declare that they have no competing interests.

\section{References}

1. Hungin APS, Whorwell PJ, Tack J, Mearin F. The prevalence, patterns and impact of irritable bowel syndrome: an international survey of 40000 subjects. Aliment Pharmacol Ther. 2003;5:643-50.

2. Philpott H, Gibson P, Thien F. Irritable bowel syndrome - an inflammatory disease involving mast cells. Asia Pac Allergy. 2011;1:36-42.

3. Canavan C, West J, Card T: The epidemiology of irritable bowel syndrome. Clin Epidemiol. 2014;6:71-80.

4. Mearin F, Badía X, Balboa A, Bero E, Caldwell E, Cucala M, et al. Irritable bowel syndrome prevalence varies enormously depending on the employed diagnostic criteria: comparison of rome II versus previous criteria in a general population. Scand $J$ Gastroenterol. 2001;36:1155-61.

5. Naeem SS, Siddiqui EU, Kazi AN, Memon AA, Khan ST, Ahmed B. Prevalence and factors associated with irritable bowel syndrome among medical students of Karachi, Pakistan: a cross-sectional study. BMC Res Notes. 2012;5:3-5.

6. Krogsgaard LR, Engsbro AL, Bytzer P. The epidemiology of irritable bowel syndrome in denmark. a population-based survey in adults $\leq 50$ years of age. Scand J Gastroenterol. 2013;48:523-9.

7. López-Colombo A, Morgan D, Bravo-González D, Montiel-Jarquín A, Méndez-Martínez S, Schmulson M. The epidemiology of functional gastrointestinal disorders in Mexico: a population-based study. Gastroenterol Res Pract. 2012;2012:1-8.

8. Ebling B, Jurcić D, Gmajnić R, Vcev A, Bilić A, Pribić S. Anthropological, demographic and socioeconomic characteristics of irritable bowel syndrome. Coll Antropol. 2011;35:513-21.

9. Husain N, Chaudhry IB, Jafri F, Niaz SK, Tomenson B, Creed F. A population-based study of irritable bowel syndrome in a non-western population. Neurogastroenterol Motil. 2008;20:1022-9.

10. Jafri W, Yakoob J, Jafri N, Islam M, Ali QM. Irritable bowel syndrome and health seeking behaviour in different communities of Pakistan. J Pak Med Assoc. 2007;57:285-7.

11. Marynowski M, Likońska A, Zatorski H, Fichna J. Role of environ- 
mental pollution in irritable bowel syndrome. World J Gastroenterol. 2015;21:11371-8.

12. Locke GR, Zinsmeister AR, Talley NJ, Fett SL, Melton LJ. Risk factors for irritable bowel syndrome: role of analgesics and food sensitivities. Am J Gastroenterol. 2000;95:157-65.

13. Burns DG. Smoking in inflammatory bowel disease and the irritable bowel syndrome. S Afr Med J. 1986, 69: 232-3.

14. Thomas GA, Rhodes J, Ingram JR. Mechanisms of disease: nicotine - a review of its actions in the context of gastrointestinal disease. Nat Clin Pract Gastroenterol Hepatol. 2005;2:536-44.

15. Shah N, Siddiqui S. An overview of smoking practices in Pakistan. Pak J Med Sci. 2015;31:467-70.

16. Canavan C, West J, Card T. Review article: the economic impact of the irritable bowel syndrome. Aliment Pharmacol Ther. 2014;40:1023-34.

17. Khademolhosseini F, Mehrabani D, Nejabat M, Behesht M, Heydari ST, Miramazizadeh A, et al. Irritable bowel syndrome in adults over 35 years in Shiraz, southern Iran: prevalence and associated factors. J Res Med Sci. 2011;16:200-6.

18. Al Saadi T, Idris A, Turk T, Alkhatib M. Epidemiology and risk factors of uninvestigated dyspepsia, irritable bowel syndrome, and gastroesophageal reflux disease among students of Damascus university, Syria. J Epidemiol Glob Health. 2016;6:285-93.

19. Farzaneh N, Ghobaklou M, Moghimi-Dehkordi B, Naderi N, Fadai F. Effects of demographic factors body mass index, alcohol drinking and smoking habits on irritable bowel syndrome: a case control study. Ann Med Health Sci Res. 2013;3:391-6.

20. Wang H, Zhao J, Hu N, Ren J, Du M, Zhu M. Side-stream smoking reduces intestinal inflammation and increases expression of tight junction proteins. World J Gastroenterol. 2012;18:2180-7.

21. Scott AM, Kellow JE, Eckersley GM, Nolan JM, Jones MP. Cigarette smoking and nicotine delay postprandial mouth-cecum transit time. Dig Dis Sci. 1992;37:1544-7.

22. Müller-Lissner SA, Kaatz V, Brandt W, Keller J, Layer P. The perceived effect of various foods and beverages on stool consistency. Eur J Gastroenterol Hepatol. 2005;17:109-12.

23. Jafri W, Yakoob J, Jafri N, Islam M, Ali QM. Frequency of irritable bowel syndrome in college students. J Ayub Med Coll Abbottabad. 2005;17:9-11.

24. Adeyemo MA, Spiegel BM, Chang L: Meta-analysis: do irritable bowel syndrome symptoms vary between men and women? Aliment Pharmacol Ther. 2010, 32: 738-55.

25. Camilleri M, Mayer EA, Drossman DA, Heath A, Dukes GE, McSorley D, et al. Improvement in pain and bowel function in female irritable bowel patients with alosetron, a 5-HT3 receptor antagonist. Aliment Pharmacol Ther. 1999;13:1149-59.

26. Böhn L, Störsrud S, Törnblom H, Bengtsson U, Simrén M. Selfreported food-related gastrointestinal symptoms in ibs are common and associated with more severe symptoms and reduced quality of life. Am J Gastroenterol. 2013;108:634-41.

27. Mönnikes H. Quality of life in patients with irritable bowel syndrome. J Clin Gastroenterol. 2011;45:98-101. 